\title{
Extraction of the resonance parameters at finite times
}

\author{
Ulf-G. Meißner a,b, Kathryn Polejaeva ${ }^{\mathrm{a}, *}$, Akaki Rusetsky ${ }^{\mathrm{a}}$ \\ ${ }^{a}$ Helmholtz-Institut für Strahlen- und Kernphysik (Theorie), and Bethe Center for Theoretical Physics, \\ Universität Bonn, 53115 Bonn, Germany \\ ${ }^{\mathrm{b}}$ Forschungszentrum Jülich, Institut für Kernphysik (IKP-3), Jülich Center for Hadron Physics \\ and Institute for Advanced Simulation (IAS-4), D-52425 Jülich, Germany
}

Received 20 July 2010; received in revised form 17 December 2010; accepted 20 December 2010

Available online 24 December 2010

\begin{abstract}
In this paper we propose a model-independent method to extract the resonance parameters on the lattice directly from the Euclidean 2-point correlation functions of the field operators at finite times. The method is tested in case of the two-point function of the $\Delta$-resonance, calculated at one loop in Small Scale Expansion. Further, the method is applied to a 1+1-dimensional model with two coupled Ising spins and the results are compared with earlier ones obtained by using Lüscher's approach.

(c) 2011 Elsevier B.V. All rights reserved.
\end{abstract}

Keywords: Unstable states; Lattice field theory; Ising model

\section{Introduction}

It is well known that the asymptotic behavior of the two-point function for large Euclidean times is determined by the lowest eigenvalue of the Hamiltonian in a given channel. In case of stable particles, this property allows one to determine their masses. The case of the excited states is different. Here, the two-point function yields the spectrum of the so-called "scattering states." The relation to the energy and width of the resonance states is not direct, since a resonance, in general, cannot be associated with an isolated energy level of a Hamiltonian. Up to now, several

\footnotetext{
* Corresponding author.

E-mail addresses: meissner@hiskp.uni-bonn.de (U.-G. Meißner), polejaeva@hiskp.uni-bonn.de (K. Polejaeva), rusetsky@hiskp.uni-bonn.de (A. Rusetsky).
} 
alternative methods have been used to determine these quantities from the lattice Monte Carlo (MC) simulations. These are:

i) At present, Lüscher's approach [1-4] is widely used to deal with the resonances in lattice QCD, obtained in simulations with sufficiently low quark masses. In brief, the procedure consists in determining first the phase shift by studying the volume dependence of the energy spectrum on the lattice. Then, continuing the $S$-matrix into the complex plane (e.g., by using the effective-range expansion whenever possible), one attempts to determine the position of the poles on the second Riemann sheet. This procedure is described in detail in Ref. [5], where the generalization to the case of the resonance matrix elements (in $1+1$ dimensions) is also considered. A shortcut is provided by using Breit-Wigner type parameterization for the scattering phase and determining its parameters (energy and width) from the lattice data (see, e.g. Ref. [6] and Refs. [7,8], where the method has been applied in the case of the $\sigma$ - and $\rho$-mesons, respectively). Lüscher's approach has been also generalized to moving frames [9].

ii) Recently, the spectral functions in QCD have been reconstructed by using the maximal entropy method (see, e.g. [10-12]). This method, as well as Lüscher's approach, has in principle the capacity to address the problem of the extraction of the resonance energy and width from the Euclidean MC simulations on the lattice.

iii) The Euclidean correlators have been parameterized in terms of the energy and width of an isolated resonance state, in order to subsequently determine these quantities from the fit to the lattice data [13]. In that paper, the method has been applied to study the glueball decay.

iv) In certain cases, the decay width of an excited state can be evaluated by calculating decay amplitudes on the lattice (see, e.g. [14,15]).

v) Recently, there has been a substantial activity in the determination of the excited meson and baryon spectrum by using generalized eigenvalue equations [16-23]. Despite spectacular progress achieved in the field, it should be stressed once again that a resonance state cannot be uniquely associated with a particular energy level. To a certain extent, excited states and scattering states can be distinguished, e.g., by studying the volume dependence of the spectral density [24,25]. This method, however works for narrow resonances only [26].

In this paper, we combine some of the above ideas and propose a systematic method to extract resonance pole positions from lattice data. In its present form, our approach is applicable to the systems with a low-lying, well-isolated, narrow resonance in the spectrum (for example, the $\rho$ or the $\Delta(1232)$ ). First, we have tested our method using synthetic input data, represented by the Euclidean propagator of the $\Delta$, calculated in the low-energy effective field theory at one loop. A further test has been carried out in a 1+1-dimensional model of two coupled Ising spins, where the resonance parameters have been determined in the past utilizing Lüscher's approach [27,28]. In both cases, we find that the method is capable to extract the pole position of the resonance.

The outline of the paper is as follows. In Section 2 we discuss the foundations of the method. The general representation of the two-point function in the presence of a low-lying isolated resonance is discussed in Section 3. In Section 4 we consider the procedure of the data fitting and the determination of the pole position by using synthetic data. A short review of the $1+1$ dimensional Ising model is given in Section 5. The extraction of the resonance pole in this model is considered in Section 6. Finally, Section 7 contains our conclusions. Some technicalities are relegated to the appendices. 


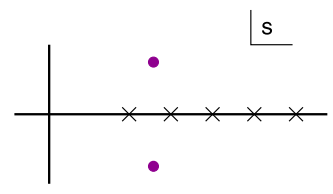

a

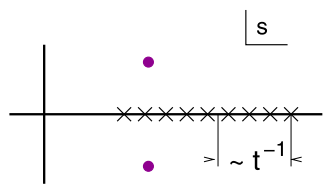

b

Fig. 1. Schematic representation of the analytic structure of the momentum-space two-point function in the rest frame $p_{\mu}=(\omega, 0)$ as a function of the complex variable $s=(i \omega)^{2}$, for two different values of the box size $L$. The crosses on the real axis denote the poles, and the shaded blobs, which are located symmetrically to the real axis, mark the location of the resonance poles on the second Riemann sheet emerging in the infinite volume limit. At any finite value of $L$, however, the two-point function is meromorphic and the second sheet does not arise. If the two-point function is measured for Euclidean times $\leqslant t$, the "energy resolution" of such a measurement is approximately equal to $t^{-1}$, as indicated on the right panel.

\section{Källen-Lehmann representation}

In the beginning of this section, we present a qualitative reasoning to justify our method. We start by mentioning that lattice QCD simulations are always carried out on lattices with a finite (Euclidean) time and spatial extension. Below, we consider lattices of the size $T \times L^{d}$, where $T$ and $L$ denote the box size in time and space, respectively, and $d$ is the number of spatial dimensions. For not so large $L$ (however, large enough to suppress the polarization effects in stable particles), the energy levels are well separated and can in principle be extracted from the asymptotic behavior of the two-point function at large Euclidean times $t \gg \Delta E$, where $\Delta E$ denotes the average level spacing. The Fourier-transform of the two-point function is a meromorphic function in the complex s-plane (see Fig. 1a). Consequently, the second Riemann sheet, as well as the poles on it (corresponding to the resonances), do not appear at any finite $L$. The information about these poles stays encoded in the dependence of the spectrum on the spatial box size $L$ and can be extracted (in several consecutive steps, as explained in the Introduction) by using Lüscher's approach.

In case an almost stable state is present, such a complicated procedure might seem superfluous. Intuitively, it is clear that, if the decay width $\Gamma$ is small, the resonance will behave pretty much the same way as a stable particle and will determine the $t$-dependence of the two-point function in a large interval (however, not for the asymptotically large times $t \gg \Gamma^{-1}$, when the resonance already has decayed). Consider, for example, a lattice with the large spatial size $L$, and calculate the two-point function at finite $t$ (see Fig. 1b). If the "resolution" $\sim t^{-1}$ is larger than the distance between energy levels, the two-point function is given by a sum of (many) exponentials with the spectral weight suppressed by a factor $L^{-d}$, so that, effectively, the spectral sum transforms into an integral over the energies. This is equivalent to the emergence of the cut that connects the physical sheet to the second sheet. We expect that, in this case, one may find an alternative representation of the two-point function in terms of quasiparticle degrees of freedom corresponding to the pole(s) on the second Riemann sheet (i.e. the resonance decay and width) plus a small background which can be described by a few parameters. Namely, we expect that there exists a window in the variable $t$, where such a description will be more effective than the multi-exponential representation through the energies and spectral weights.

Hence, the original problem is reduced to finding a universal, model-independent parameterization of the two-point function in the presence of a narrow resonance, which will allow one to determine the energy and the width of the latter by performing a fit to the lattice data. This is analogous to the exponential parameterization, which allows one to determine the mass of a 
stable particle by fitting at asymptotically large times. Note that we shall try to avoid approximations in the spectral function (e.g. the narrow width approximation used in Ref. [13]), which are a potential source of a systematic error. The contribution of the background, albeit small, will be taken into account in a systematic manner.

The model-independent parameterization, which was mentioned above, can be obtained directly by using the Källen-Lehmann representation for the two-point function. Below, we give a brief derivation of this representation in a finite box. Further, we consider the limits $T \rightarrow \infty$ and $L \rightarrow \infty$ in detail, in order to quantify the qualitative arguments given in the beginning of this section.

In the derivation of the Källen-Lehmann representation in a finite volume, we mainly follow the steps given in Ref. [10]. Let $\phi(x) \doteq \phi(t, \mathbf{x})$ be any local field, carrying the resonance quantum numbers. The two-point function of this field in the Euclidean space is defined as

$$
\begin{aligned}
& D(t, \mathbf{x})=\left\langle N_{t}\left[\phi(t, \mathbf{x}) \phi^{\dagger}(0)\right]\right\rangle=\frac{1}{Z(T)} \operatorname{Tr}\left(N_{t}\left[\phi(t, \mathbf{x}) \phi^{\dagger}(0)\right] \mathrm{e}^{-H T}\right), \\
& N_{t}\left[\phi(t, \mathbf{x}) \phi^{\dagger}(0)\right] \doteq \theta(t) \phi(t, \mathbf{x}) \phi^{\dagger}(0) \pm \theta(-t) \phi^{\dagger}(0) \phi(t, \mathbf{x}), \\
& Z(T)=\operatorname{Tr}\left(\mathrm{e}^{-H T}\right),
\end{aligned}
$$

where the upper (lower) sign corresponds to bosons (fermions). The Fourier transform of this expression takes the form

$$
D(t, \mathbf{x})=\frac{1}{T L^{d}} \sum_{\omega} \sum_{\mathbf{k}} D(i \omega, \mathbf{k}) \mathrm{e}^{-i \omega t-i \mathbf{k x}}
$$

where $\omega=\frac{2 \pi}{T} n_{T}\left(\omega=\frac{\pi}{T}\left(2 n_{T}+1\right)\right)$, with $n_{T} \in \mathbb{Z}$, are Matsubara frequencies in case of bosons (fermions), and $\mathbf{k}=\frac{2 \pi}{L} \mathbf{n}$ with $\mathbf{n} \in \mathbb{Z}^{d}$.

Using a complete set of Hamiltonian eigenvectors $H|\alpha\rangle=E_{\alpha}|\alpha\rangle$ to calculate the trace in Eq. (1), one gets

$$
D(i \omega, \mathbf{k})=-\frac{L^{d}}{Z(T)} \sum_{\alpha} \sum_{\beta} \delta_{\mathbf{k}, \mathbf{p}_{\beta}-\mathbf{p}_{\alpha}} \frac{\mathrm{e}^{-E_{\alpha} T} \mp \mathrm{e}^{-E_{\beta} T}}{E_{\alpha}-E_{\beta}+i \omega}\langle\alpha|\phi(0)| \beta\rangle\left\langle\beta\left|\phi^{\dagger}(0)\right| \alpha\right\rangle,
$$

where $\delta_{\mathbf{k}, \mathbf{q}}^{d}$ denotes the periodic Kronecker $\delta$ in $d$ dimensions. This relation can be rewritten as

$$
D(i \omega, \mathbf{k})=\int_{-\infty}^{\infty} \frac{d \omega^{\prime}}{\omega^{\prime}-i \omega} A\left(\omega^{\prime}, \mathbf{k}\right)
$$

where the spectral function is given by

$$
\begin{aligned}
A\left(\omega^{\prime}, \mathbf{k}\right)= & \frac{L^{d}}{Z(T)} \sum_{\alpha} \sum_{\beta}\left(\mathrm{e}^{-E_{\alpha} T} \mp \mathrm{e}^{-E_{\beta} T}\right) \delta\left(\omega^{\prime}-E_{\beta}+E_{\alpha}\right) \\
& \times \delta_{\mathbf{k}, \mathbf{p}_{\beta}-\mathbf{p}_{\alpha}}^{d}\langle\alpha|\phi(0)| \beta\rangle\left\langle\beta\left|\phi^{\dagger}(0)\right| \alpha\right\rangle .
\end{aligned}
$$

By applying discrete symmetries, it can be shown that the spectral function obeys the following properties [10]

$$
A\left(\omega^{\prime}, \mathbf{k}\right) \geqslant 0 \quad \text { for } \omega^{\prime} \geqslant 0, \quad A\left(-\omega^{\prime},-\mathbf{k}\right)=\mp A\left(\omega^{\prime}, \mathbf{k}\right)=\mp A\left(\omega^{\prime},-\mathbf{k}\right) .
$$


The dispersion integral can be rewritten as ${ }^{1}$

$$
D(i \omega, \mathbf{k})=\int_{0}^{\infty} \frac{d \omega^{\prime 2}}{\omega^{\prime 2}+\omega^{2}} A\left(\omega^{\prime}, \mathbf{k}\right)
$$

In the limit $T \rightarrow \infty$, only the vacuum state $\alpha=0, E_{\alpha}=0$ contributes, and the spectral function in Eq. (7) is given by

$$
\lim _{T \rightarrow \infty} A\left(\omega^{\prime}, \mathbf{k}\right)=L^{d} \sum_{\beta} \delta\left(\omega^{\prime}-E_{\beta}\right) \delta_{\mathbf{k}, \mathbf{p}_{\beta}}^{d}|\langle 0|\phi(0)| \beta\rangle|^{2} .
$$

It is seen that in the limit $T \rightarrow \infty$ the integral in Eq. (7) is performed from $\omega_{\min }^{2}$ to infinity, where $\omega_{\min }$ is determined by the lowest-mass intermediate state in a finite volume.

Let us now discuss the infinite-volume limit in the two-point function. ${ }^{2}$ Set $\mathbf{k}=0$ and enumerate the singularities $-\omega_{i}^{2}$ with $i=1,2, \ldots$ with $-\omega_{1}^{2}=\omega_{\min }^{2}$. For each $i$ define an open disc $d_{i}$ in a complex plane

$$
\forall i, \quad d_{i}=\left\{z \in \mathbb{C}|| \operatorname{Re} z-\omega_{i}^{2}\left|<\operatorname{Re} h_{i},\right| \operatorname{Im} z-\omega_{i}^{2} \mid<\operatorname{Im} h_{i}\right\},
$$

where $h_{i}=\Delta_{i}+i \varepsilon, \Delta_{i}$ is the distance between the neighboring poles $\Delta_{i}=\left|\omega_{i+1}^{2}-\omega_{i}^{2}\right|$, and $\varepsilon>0$ is a fixed constant. Then, the domain $\mathcal{D}=\bigcup_{i} d_{i}$ defines a narrow strip along the negative real axis.

Assume now that $\omega^{2} \in \mathbb{C} \backslash \mathcal{D}$ and consider a generic Feynman graph in a finite volume

$$
I\left(\omega^{2}\right)=\prod_{i} \frac{1}{L^{d}} \sum_{\mathbf{l}_{i}} J\left(\omega^{2}, \mathbf{l}_{i}\right), \quad J\left(\omega^{2}, \mathbf{l}_{i}\right)=\prod_{i} \int \frac{d l_{i}^{0}}{2 \pi} \prod_{k} \frac{1}{q_{k}^{2}+m_{k}^{2}},
$$

where $l_{i}$ denote the loop momenta and $q_{k}=a_{k} P+\sum_{i} b_{k i} l_{i}$ are the momenta corresponding to an internal line, which are linear combinations of the loop momenta and the external momentum $P^{\mu}=(i \omega, \mathbf{0})$. Finally, $m_{k}$ denote the masses of particles in the loop.

In the $\mathrm{CM}$ frame each propagator in the loop can be rewritten as

$$
\frac{1}{q_{k}^{2}+m_{k}^{2}}=\frac{1}{\left(q_{k}^{0}\right)^{2}+w_{k}^{2}}, \quad w_{k}^{2}=m_{k}^{2}+\left(\sum_{i} b_{k i} \mathbf{l}_{i}\right)^{2} .
$$

It is easy to see that $J\left(\omega^{2}, \mathbf{l}_{i}\right)$ is differentiable with respect to $\mathbf{l}_{i}$ and that the differentiation does not change its analytic properties. From Eqs. (10), (11) it is also seen that $J\left(\omega^{2}, \mathbf{l}_{i}\right)$, as a function of $\omega^{2}$, is analytic on the complex plane outside the strip. Then, $J\left(\omega^{2}, \mathbf{l}_{i}\right)$ is a continuous function of 3-momenta $\mathbf{l}_{i}$ outside the strip and the regular summation theorem [30] can be applied. Finally, we conclude that,

if the variable $\omega^{2}$ lies outside the strip, the function $D(i \omega)$ approaches its infinite-volume limit $D^{\infty}(i \omega, \mathbf{k})$ faster than any power of $L$.

Moreover, the function $2 \omega^{\prime}\left(\omega^{\prime 2}+\omega^{2}\right)^{-1}$ in the interval $\omega_{0}-\Delta / 2<\omega^{\prime}<\omega_{0}+\Delta / 2$ for all $\Delta \geqslant 0, \omega_{0}-\Delta / 2 \geqslant \omega_{\min }$ is bound from above by some large constant $B=B\left(\omega_{0}, \Delta, \omega^{2}\right)$. From this we get

\footnotetext{
1 For illustrative purpose, below we display the bosonic case only. The fermionic case can be treated similarly.

2 for a related discussion, see also Ref. [29].
} 


$$
\left|D(i \omega, \mathbf{k})-D^{\infty}(i \omega, \mathbf{k})\right| \leqslant B \int_{\omega_{0}-\Delta / 2}^{\omega_{0}+\Delta / 2} d \omega^{\prime}\left|A\left(\omega^{\prime}, \mathbf{k}\right)-A^{\infty}\left(\omega^{\prime}, \mathbf{k}\right)\right|,
$$

where the infinite-volume spectral function is defined as

$$
A^{\infty}\left(\omega^{\prime}, \mathbf{k}\right)=\sum_{\beta} \delta\left(\omega^{\prime}-E_{\beta}\right)(2 \pi)^{d} \delta^{d}\left(\mathbf{k}-\mathbf{p}_{\beta}\right)|\langle 0|\phi(0)| \beta\rangle|^{2} .
$$

Here, $\mathbb{Z}_{\beta}$ stands for the sum (integral) over the continuous spectrum wave functions.

Since the left-hand side of Eq. (12) exponentially vanishes for a large $L$, the right-hand side must do the same. In conclusion, for $\omega^{2}$ outside the strip, the difference $A\left(\omega_{0}, \Delta, \mathbf{k}\right)-$ $A^{\infty}\left(\omega_{0}, \Delta, \mathbf{k}\right)$, where

$$
A\left(\omega_{0}, \Delta, \mathbf{k}\right)=\int_{\omega_{0}-\Delta / 2}^{\omega_{0}+\Delta / 2} d \omega^{\prime} A\left(\omega^{\prime}, \mathbf{k}\right), \quad A^{\infty}\left(\omega_{0}, \Delta, \mathbf{k}\right)=\int_{\omega_{0}-\Delta / 2}^{\omega_{0}+\Delta / 2} d \omega^{\prime} A^{\infty}\left(\omega^{\prime}, \mathbf{k}\right),
$$

for any $\omega_{0}>\omega_{\min }$ and $\Delta>0$ converges faster than any power of $L$ as $L \rightarrow \infty$. This statement is a mathematical formulation for the intuitive picture of "poles merging into the cut," which is shown in Fig. $1 b$.

We further illustrate the limit $L \rightarrow \infty$ with two examples. First, in order Eq. (14) to hold, the matrix elements $\langle 0|\phi(0)| \beta\rangle$ should be continuous functions of $E_{\beta}$ at a finite $L$. We examine these matrix elements explicitly in a simple quantum mechanical model in Appendix A and explicitly check that the above requirement is indeed fulfilled. Further, in Appendix B we give an example of a function which is meromorphic at a finite $L$ and develops a cut and a pole on the second Riemann sheet in the limit $L \rightarrow \infty$. This example models the behavior of the function $D(i \omega)$.

Performing the Fourier transform, from Eq. (2) one finds

$$
\begin{aligned}
D(t, \mathbf{k})=\frac{1}{T} \sum_{\omega} \mathrm{e}^{-i \omega t} D(i \omega, \mathbf{k}) & =\int_{\omega_{\min }}^{\infty} d \omega^{\prime} \frac{\mathrm{e}^{-\omega^{\prime}(T-t)}+\mathrm{e}^{-\omega^{\prime} t}}{1-\mathrm{e}^{-\omega^{\prime} T}} A\left(\omega^{\prime}, \mathbf{k}\right) \\
& \rightarrow \int_{\omega_{\min }}^{\infty} d \omega^{\prime} \mathrm{e}^{-\omega^{\prime} t} A\left(\omega^{\prime}, \mathbf{k}\right) .
\end{aligned}
$$

The last line is obtained in the limit $T \rightarrow \infty$. Together with the expression (8) for the spectral density, we recover the representation for $D(t, \mathbf{k})$ as a sum over exponentials. In the case $t^{-1}$ is much larger than the distance between different energy levels (this can be achieved, e.g., by holding $t$ fixed and increasing $L)$, many exponentials contribute to $D(t, \mathbf{k})$ and the sum over the energy eigenvalues can be replaced through the integral. In this case, $A\left(\omega^{\prime}, \mathbf{k}\right)$ is replaced by $A^{\infty}\left(\omega^{\prime}, \mathbf{k}\right)$.

To summarize, the behavior of the two-point function can be studied in different regimes. For asymptotically large $t$ and moderately large $L$, only the few lowest, well-separated energy levels contribute. This situation is well described by a sum of a few exponential terms. In contrast to this, in the regime with asymptotically large $L$ and moderately large $t$ there are many terms with nearly the same energies that contribute to the multi-exponential representation. The sum over the discrete energy spectrum effectively transforms into an integral. If, in addition, a lowlying well-separated resonance emerges, we expect that the spectral integral can be efficiently parameterized in terms of the resonance parameters instead of the stable energy levels. 


\section{Two-point function at finite $t$}

As discussed in the previous section, it is possible to perform the infinite-volume limit $L \rightarrow \infty$ in the two-point function, keeping the Euclidean time $t$ fixed. The spectral representation is given by Eq. (7) with the spectral density given by Eq. (13). Note that the spectral density vanishes for $0 \leqslant \omega^{\prime} \leqslant w_{\min }$, and hence the integration in Eq. (7) in fact is performed from $\omega^{\prime 2}=\omega_{\min }^{2}$ to infinity.

For simplicity, we work in the center-of-mass $(\mathrm{CM})$ frame $\mathbf{k}=0$ and denote $D(i \omega, \mathbf{0}) \doteq$ $D(i \omega), A^{\infty}\left(\omega^{\prime}, \mathbf{0}\right) \doteq A\left(\omega^{\prime}\right)$. The spectral representation then takes the form

$$
D(i \omega)=\int_{\omega_{\min }}^{\infty} \frac{2 \omega^{\prime} d \omega^{\prime}}{\omega^{\prime 2}+\omega^{2}} A\left(\omega^{\prime}\right) .
$$

In the vicinity of the elastic threshold, $A\left(\omega^{\prime}\right) \sim\left(\omega^{\prime}-\omega_{\min }\right)^{l+1 / 2}$, where $l$ stands for the orbital angular momentum. ${ }^{3}$

Assume now that an isolated low-lying resonance emerges. This is equivalent to the statement that the function $A\left(\omega^{\prime}\right)$ takes the form

$$
A\left(\omega^{\prime}\right)=\frac{\left(\omega^{\prime}-\omega_{\min }\right)^{l+1 / 2}}{\left(\omega^{\prime}-\omega_{R}\right)\left(\omega^{\prime}-\omega_{R}^{*}\right)} Q\left(\omega^{\prime}\right),
$$

where the singularities of the function $Q\left(\omega^{\prime}\right)$ lie far enough from the threshold so that the Taylor expansion of this function converges in the part of the complex plane that includes the resonance poles at $\omega^{\prime}=\omega_{R}$ and $\omega^{\prime}=\omega_{R}^{*}$. The energy and the width of the resonance is determined by $\omega_{R}$ in the standard manner. Note that these poles come in complex-conjugated pairs. ${ }^{4}$

Using Eq. (17), it can be easily shown that

$$
D(t)=\int_{\omega_{\min }}^{\infty} d \omega^{\prime} \mathrm{e}^{-\omega^{\prime} t} A\left(\omega^{\prime}\right)=\mathrm{e}^{-\omega_{\min } t} \int_{0}^{\infty} \frac{d E E^{l+1 / 2} \mathrm{e}^{-E t}}{\left(E-E_{R}\right)\left(E-E_{R}^{*}\right)} Q\left(E+\omega_{\min }\right),
$$

where $E_{R}=\omega_{R}-\omega_{\min }=E_{0}-i \Gamma / 2$. As mentioned before, it is assumed that the Taylor expansion $Q\left(E+\omega_{\min }\right)=\sum_{k=0}^{\infty} q_{k} E^{k}$ converges in the part of a complex region which includes the resonance poles.

From the above expression we get

$$
D(t)=\mathrm{e}^{-\omega_{\min } t} \sum_{k=0}^{\infty} q_{k} F^{(k+l)}\left(t, E_{R}\right), \quad F^{(m)}\left(t, E_{R}\right)=\int_{0}^{\infty} \frac{d E E^{m+1 / 2} \mathrm{e}^{-E t}}{\left(E-E_{0}\right)^{2}+\Gamma^{2} / 4} .
$$

In particular, for $m=0,1$, we find

$$
\begin{aligned}
& F^{(0)}\left(t, E_{R}\right)=-\frac{2}{\Gamma} \operatorname{Im} \chi\left(t, E_{R}\right), \\
& F^{(1)}\left(t, E_{R}\right)=\operatorname{Re} \chi\left(t, E_{R}\right)-\frac{2 E_{0}}{\Gamma} \operatorname{Im} \chi\left(t, E_{R}\right),
\end{aligned}
$$

\footnotetext{
3 This statement is valid in $3+1$ dimensions. In $1+1$ dimensions, one has to substitute $l=0$ in all formulae.

$4 A\left(\omega^{\prime}\right)$ is the discontinuity of a function which is analytic in the cut complex plane and obeys Schwarz reflection principle. Hence the poles in this function (which emerge on the second Riemann sheet), always come in pairs. This is the justification for the ansatz (17).
} 


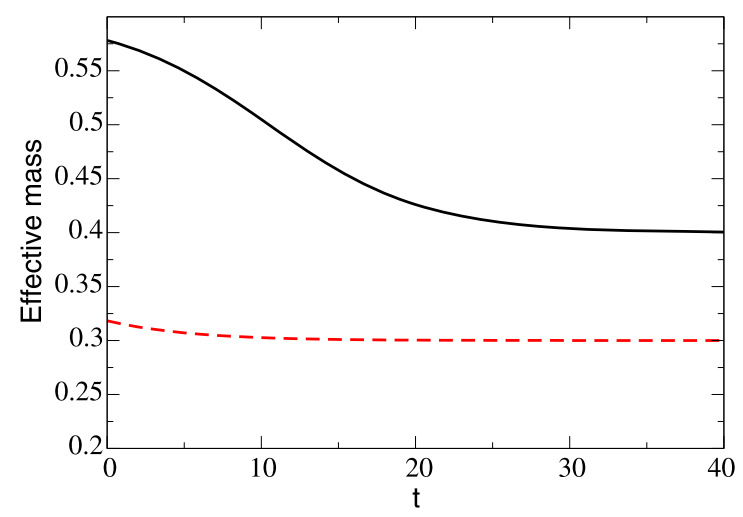

Fig. 2. Schematic representation of the effective mass for a stable particle (dashed line) and for a resonance (solid line).

where

$$
\chi\left(t, E_{R}\right)=\int_{0}^{\infty} \frac{d E E^{1 / 2} \mathrm{e}^{-E t}}{E-E_{R}}
$$

and the following representation in form of an infinite series is useful in a wide range of the variable $t$

$$
\chi\left(t, E_{R}\right)=-\pi \sqrt{-E_{R}} \mathrm{e}^{-E_{R} t}+\sqrt{\frac{\pi}{t}}\left\{1+\sum_{k=0}^{\infty} \frac{\left(-2 E_{R} t\right)^{k+1}}{(2 k+1) ! !}\right\} .
$$

Further, the functions $F^{(m)}$ with $m \geqslant 2$ can be recursively expressed through $F^{(0,1)}$. The general representation of the two-point function follows straightforwardly from the above relations

$$
D(t)=\mathrm{e}^{-\omega_{\min } t}\left\{c_{0} F^{(0)}\left(t, E_{R}\right)+c_{1} F^{(1)}\left(t, E_{R}\right)+\sum_{k=0}^{\infty} \frac{x_{k}}{t^{l+k+3 / 2}}\right\},
$$

where $c_{0,1}$ and $x_{k}$ are expressed through the Taylor coefficients $q_{k}$ as well as $E_{0}, \Gamma$.

Eq. (23) represents our central result. It provides a universal parameterization of the Euclidean two-point function in the presence of a low-lying isolated resonance described by two parameters $E_{0}, \Gamma$. The couplings $x_{k}$ are associated with the non-resonant background. In particular, it encodes the contribution of the threshold which lies below the resonance energy. This means that if $t$ is taken too large, the background dominates and the information about $E_{0}, \Gamma$ is erased. We assume, however, that in the presence of a narrow resonance, there exists a sufficiently wide window in $t$, where the background is small and $E_{0}, \Gamma$ can be determined from the fit of the measured $D(t)$ to the representation (23). We require that, in this window, adding the background parameterized by the constants $x_{k}$ should lead to small corrections in $E_{0}, \Gamma$ and the fit should remain stable against the increase of the number of independent $x_{k}$.

The physical meaning of our method can be easily illustrated by Fig. 2. In this figure, the effective mass of a system in the presence of a stable state/resonance is schematically depicted. If there is a stable particle, the plateau in the effective mass sets in almost immediately. However, if a resonance instead of a stable particle is present, there exists a wide window in $t$, where many excited states contribute and the effective mass decreases slowly until it reaches the asymptotic 
value. Our method roughly corresponds to fitting $D(t)$ within this interval by the representation given in Eq. (23), and the decay width is determined by the rate of the decrease of the effective mass. Note that a similar picture was obtained in Ref. [31]. In that paper, the theory of two coupled scalar fields, where the heavier field can decay into a couple of light scalars, was considered. In particular, it has been shown that the effective mass of the heavy scalar, calculated on the lattice, exhibits the same behavior when its mass is below/above the two-particle threshold (see Fig. 1 of that article). The two point functions of the excited mesons in QCD also exhibit a similar behavior [16].

It is instructive to compare the parameterization (23) to the pertinent formula obtained in Ref. [13]. A technical difference consists in the absence of the threshold factor $\left(\omega^{\prime}-\omega_{\min }\right)^{l+1 / 2}$ that results in a different parameterization of the background. The important difference is, however, that in Ref. [13] a Breit-Wigner type parameterization for the spectral function is originally assumed. Since this ansatz did not fit the lattice data well, an ad hoc energy-dependence in the decay width has been introduced, and the functional form of this dependence has been determined by using a trial-and-error method. In our approach, the representation of $D(t)$, given in Eq. (23) is completely general and is based on the sole assumption that a well-isolated resonance emerges at low energies. The background is parameterized by the constants $x_{k}$ in a systematic manner.

\section{The fit}

We first test our method by using synthetic data. Consider, for instance, the propagator of the $\Delta$-resonance evaluated in the Small Scale Expansion $(\mathrm{SSE})^{5}$ at one loop [33,34]. In Minkowski space, this propagator is given by

$$
\begin{aligned}
& S_{\Delta}(p)=-S_{\Delta}^{3 / 2}(p) \Pi^{3 / 2}+\text { spin-1/2, } \\
& S_{\Delta}^{3 / 2}(p)=\frac{1}{\dot{m}_{\Delta}\left(1+\Sigma_{2}\left(p^{2}\right)\right)-\not p\left(1-\Sigma_{1}\left(p^{2}\right)\right)},
\end{aligned}
$$

where $\stackrel{\circ}{m}_{\Delta}$ denotes the mass of the $\Delta$-resonance in the chiral limit, $\Pi^{3 / 2}$ stands for the projector onto the spin-3/2 state and the spin-1/2 part does not have a pole in the low-energy region (for a general proof of this statement, see [35]). Further, the invariant functions $\Sigma_{1,2}\left(p^{2}\right)$ at order $p^{3}$ in the chiral expansion are given by

$$
\Sigma_{1}(s)=\frac{c_{A}^{2}}{F^{2}}\left(W_{2}(s)-W_{3}(s)\right), \quad \Sigma_{2}(s)=\frac{c_{A}^{2}}{F^{2}} \frac{m_{N}}{m_{\Delta}} W_{2}(s),
$$

where $c_{A}$ and $F$ denote the $\pi N \Delta$ coupling constant and the pion decay constant in the chiral limit, respectively, $m_{N}, m_{\Delta}, M_{\pi}$ are the nucleon, $\Delta$ and pion masses, respectively, and the invariant functions $W_{2,3}$ are given by $[33,34]$

$$
W_{3}(s)=\frac{-s+m_{N}^{2}-M_{\pi}^{2}}{-2 s} W_{2}(s)+\frac{M_{\pi}^{4}}{128 \pi^{2} s}\left(\ln \frac{M_{\pi}^{2}}{m_{N}^{2}}+\frac{1}{6}\right),
$$

\footnotetext{
5 The SSE is a phenomenological extension of Chiral Perturbation Theory in which the $\Delta$-nucleon mass splitting is counted as an additional small parameter. This quantity, however, does not vanish in the chiral limit. The framework of the SSE is laid in detail in Ref. [32].
} 


$$
\begin{aligned}
& W_{2}(s)=-\frac{1}{12 s}\left(\lambda W_{0}(s)-\left(-s+m_{N}^{2}-M_{\pi}^{2}\right) \frac{M_{\pi}^{2}}{16 \pi^{2}} \ln \frac{M_{\pi}^{2}}{m_{N}^{2}}\right), \\
& W_{0}(s)=\frac{i \sqrt{\lambda}}{16 \pi s}-\frac{s-m_{N}^{2}+M_{\pi}^{2}}{32 \pi^{2} s}\left(\ln \frac{M_{\pi}^{2}}{m_{N}^{2}}-1\right)-\frac{\sqrt{\lambda}}{32 \pi^{2} s} \ln \frac{s+M_{\pi}^{2}-m_{N}^{2}+\sqrt{\lambda}}{s+M_{\pi}^{2}-m_{N}^{2}-\sqrt{\lambda}}, \\
& \lambda=\left(s-\left(m_{N}+M_{\pi}\right)^{2}\right)\left(s-\left(m_{N}-M_{\pi}\right)^{2}\right) .
\end{aligned}
$$

The trace of $S_{\Delta}^{3 / 2}(p)$ obeys the dispersion relation

$$
\begin{aligned}
\operatorname{Tr} S_{\Delta}^{3 / 2}(p) & =\frac{4 \stackrel{\circ}{\Delta}_{\Delta}\left(1+\Sigma_{2}\left(p^{2}\right)\right)}{\left(\dot{m}_{\Delta}\left(1+\Sigma_{2}\left(p^{2}\right)\right)\right)^{2}-p^{2}\left(1-\Sigma_{1}\left(p^{2}\right)\right)^{2}} \\
& =\int_{\left(m_{N}+M_{\pi}\right)^{2}}^{\infty} \frac{d s^{\prime}}{s^{\prime}-p^{2}-i \epsilon} A\left(s^{\prime}\right),
\end{aligned}
$$

where the expression for the discontinuity can be directly read off from Eqs. (24)-(26).

In the calculations we have used the following values of the parameters: $m_{N}=940 \mathrm{MeV}$, $M_{\pi}=140 \mathrm{MeV}, m_{\Delta}=\stackrel{\circ}{m}_{\Delta}=1232 \mathrm{MeV}, F=F_{\pi}=92.4 \mathrm{MeV}$ and $c_{A}=1.5$ (this value leads to the width $\Gamma=124 \mathrm{MeV}$ in a $O\left(\epsilon^{3}\right)$ calculation at $\left.p^{2}=m_{\Delta}^{2}\right)$. It is easy to check that the propagator has a pole at $m_{R}=1212 \mathrm{MeV}$ and $\Gamma=76 \mathrm{MeV}$ (note the large shift in the quantity $\Gamma$ as compared to its value obtained at $p^{2}=m_{\Delta}^{2}$ that presumably is an artefact of the $O\left(\epsilon^{3}\right)$ approximation).

Next, we wish to investigate whether it is possible to recover this result by applying our method. To this end, we analytically continue Eq. (27) into Euclidean space and perform the Fourier transform with respect to the fourth component of the momentum. The resulting values are treated as synthetic data. We choose the interval $1.7 M_{\pi}^{-1}<t<4 M_{\pi}^{-1}$ and perform a least squares fit of these data to the formula (23) (the data points are assumed to be distributed equidistantly in this interval). ${ }^{6}$

In the fit, we cut the sum in Eq. (23) at some value $k_{\max }$. The fit of the 7 data points with $k_{\max }=0$ yields $m_{R}=1213 \mathrm{MeV}$ and $\Gamma=74 \mathrm{MeV}$ that is already close to the exact values. The procedure converges rapidly. At the accuracy of the digits displayed, the exact result is obtained for $k_{\max }=2$. Adding more terms, it is possible to improve the agreement with the exact result up to very many decimal digits.

To summarize, using synthetic data, we have demonstrated that our method is capable to reconstruct the exact position of a pole in a complex plane from a limited data sample. To perform a similar analysis for real Monte Carlo data is much more challenging. One of the main problems that we have encountered there, is related to the instability of the fit when $k_{\max }$ increases (this problem already arises for relatively small $k_{\max }=3$ or 4 ). Namely, the constants $x_{k}$, which describe the background, become very large in magnitude having alternating signs and this destabilizes the values of $E_{0}, \Gamma$ extracted from the fit.

In order to circumvent this problem, we have performed a Bayesian fit to the lattice MC data. A detailed description of the Bayesian fit techniques, which is well suited for our purposes, can be found, e.g. in Ref. [36]. We shall present a brief summary of the method below. The function to be minimized in the standard least squares fit is given by

\footnotetext{
6 The above particular values for the upper and lower bounds of the fitting interval are taken for the illustrative purposes only. One may, e.g., significantly reduce the upper limit without changing the final result.
} 


$$
\chi^{2}=\sum_{i}\left(D\left(t_{i}, E_{0}, \Gamma, c_{1}, c_{2}, x_{k}\right)-\bar{D}\left(t_{i}\right)\right)^{2}
$$

where $\bar{D}\left(t_{i}\right)$ are data corresponding to the points $t_{i}$. In Eq. (28) it is implicitly assumed that the MC errors in the data $\bar{D}\left(t_{i}\right)$ do not vary much with $t_{i}$. Note that the above form still does not include our prior knowledge about $x_{k}$. The assumption about the smoothness of the function $Q\left(\omega^{\prime}\right)$ in Eq. (17) implies that $x_{k}$ should be of "natural size" excluding the scenario where the $x_{k}$ become large with alternating signs.

In order to implement this prior knowledge into the fitting procedure, in analogy with Ref. [36], we define the augmented $\chi^{2}$

$$
\chi_{\text {aug }}^{2}=\chi^{2}+\chi_{\text {prior }}^{2}, \quad \chi_{\text {prior }}^{2}=\frac{1}{S^{2}} \sum_{k=0}^{k_{\max }} x_{k}^{2},
$$

where $S$ is some scale that ensures that all $x_{k}$ stay in the "natural" range.

We determine the quantity $S$ by using the trial-and-error method. If $S$ is too large, the introduction of $\chi_{\text {aug }}^{2}$ does not cure the problem with the convergence. This sets the upper limit on the value of $S$. The lower limit for $S$ is set by the requirement that the results obtained with standard $\chi^{2}$ and $\chi_{\text {aug }}^{2}$ agree for low $k_{\max }=1,2$. In addition, within this range, the final result of the fit for $E_{0}, \Gamma$ should not depend on $S$.

In the $1+1$-dimensional model with two Ising spins discussed in the next section, we have performed fits using $\chi_{\text {aug }}^{2}$. Below we show that this technique allows one to extract the precise values of $E_{0}, \Gamma$ from the lattice MC data in this model.

\section{1 + 1-dimensional model with two coupled Ising spins}

In this section we apply our method to the extraction of the resonance pole position to a $1+1$ dimensional model of two coupled Ising spins. This model has been treated in Refs. [27,28] using Lüscher's approach. In particular, it has been shown that a narrow resonance emerges in the system, whose parameters can be extracted in a systematic manner.

The action of the model is given by

$$
\begin{aligned}
S= & -\kappa_{\phi} \sum_{z \in \Lambda, \hat{\mu}=1,2} \phi_{z} \phi_{z+\hat{\mu}}-\kappa_{\eta} \sum_{z \in \Lambda, \hat{\mu}=1,2} \eta_{z} \eta_{z+\hat{\mu}} \\
& +\frac{g}{2} \sum_{z \in \Lambda, \hat{\mu}=1,2} \eta_{z} \phi_{z}\left(\phi_{z-\hat{\mu}}+\phi_{z+\hat{\mu}}\right),
\end{aligned}
$$

where $\phi_{z}, \eta_{z}= \pm 1$ are two Ising spins which interact with each other through the Yukawa-type coupling $g \eta \phi \phi$. The sum $z \in \Lambda$, where $z=(x, t)$, runs over all lattice points and $\hat{\mu}$ denotes the unit vector along the spatial axis. The couplings $\kappa_{\phi}, \kappa_{\eta}>0$ are chosen so that the masses of $\phi$ and $\eta$ are $m_{\phi} \simeq 0.19$ and $m_{\eta} \simeq 0.5$ (in lattice units). Note that, if $g \neq 0$, the $\eta$ decays into $2 \phi$, so $m_{\eta}$ corresponds to the resonance energy in this case.

The model has been analyzed in detail in Refs. [27,28]. We give only a short summary of this analysis here. In particular, it has been argued that in the theory described by the Lagrangian (30) no second-order phase transition occurs and thus the continuum limit cannot be performed. In other words, all results obtained here refer to the effective theory with an ultraviolet cutoff.

The energy spectrum is determined by solving the generalized eigenvalue problem. The operator basis is defined as in Refs. [27,28] 


$$
\mathcal{O}_{1}(t)=\frac{1}{L} \sum_{x} \eta_{x, t}, \quad \mathcal{O}_{j}(t)=\frac{1}{L^{2}} \sum_{x y} \phi_{x, t} \phi_{y, t} \mathrm{e}^{-i p_{j}(x-y)}, \quad p_{j}=\frac{2 \pi(j-2)}{L},
$$

with $j=2,3, \ldots$ The correlator matrix is given by

$$
C_{i j}(t)=\left\langle\mathcal{O}_{i}(t) \mathcal{O}_{j}(0)\right\rangle-\left\langle\mathcal{O}_{i}(t)\right\rangle\left\langle\mathcal{O}_{j}(0)\right\rangle
$$

The spectral decomposition of $C_{i j}(t)$ is approximated by the truncated series

$$
C_{i j}(t)=\sum_{l=1}^{r} v_{i}^{(l)} v_{j}^{(l) *} \mathrm{e}^{-W_{l} t}
$$

The energy eigenvalues $W_{l}$ for $l=1, \ldots, r$ are determined by diagonalizing the matrix $M\left(t, t_{0}\right) \doteq C^{-1 / 2}\left(t_{0}\right) C(t) C^{-1 / 2}\left(t_{0}\right)$, where $t_{0}$ is some fixed time (in the following, as in Refs. [27,28], we always use $\left.t_{0}=1\right)$. The eigenvalue equation takes the form

$$
M\left(t, t_{0}\right) u^{(l)}=\lambda^{(l)}\left(t, t_{0}\right) u^{(l)}, \quad \lambda^{(l)}\left(t, t_{0}\right)=\mathrm{e}^{-W_{l}\left(t-t_{0}\right)}, l=1, \ldots, r,
$$

where $u^{(l)}$ form an orthonormal basis. The eigenvectors $v^{(l)}$ are given by $v^{(l)}=\exp \left(W_{l} t / 2\right) \times$ $C^{1 / 2}\left(t_{0}\right) u^{(l)}$.

The MC simulation is done by using a cluster algorithm [37]. We closely followed the procedure described in [27,28] and, using the parameter set $\kappa_{\phi}=0.3700, \kappa_{\eta}=0.3700, g=0.04$, have reproduced the $L$-dependent spectrum calculated in this paper. The resonance parameters found in Refs. [27,28] are: $m_{\eta}=0.5112(3)$ and $\Gamma_{\eta}=0.0100(3)$. It remains to be seen, whether the same result can be obtained by using our approach.

\section{Results}

In order to use our method, one has to calculate the two-point function within a sufficiently large interval in the Euclidean time $t$ and then fit the result with Eq. (23). To this end, the correlator $C_{11}(t)$ has been chosen, see Eq. (32). However, as already mentioned in [27,28], the simulations become unstable already at $t \simeq t_{\text {unst }}=5-8$, depending on the value of $L$ chosen. The statistical error in the effective mass of $\eta$ at $t>t_{\text {unst }}$ blows up, rendering an accurate fit impossible. The use of improved estimators or a substantial increase of the number of configurations results only in a moderate improvement of the error in $C_{11}(t)$.

As described above, the energy spectrum of the system can be determined with high accuracy from the correlator matrix $C_{i j}(t)$ at $t \leqslant t_{\text {unst }}$ by applying the generalized eigenvalue method. In addition to the ground state, the approach allows a reliable extraction of higher excited levels (up to 4 or 5 levels, depending on $L$ ). The physical reason for this is that the matrix $C_{i j}(t)$ contains much more information about the system than the single function $C_{11}(t)$. In particular, it contains information about the matrix elements describing the transitions between various energy levels.

So, it is not surprising that using this input in our method helps to reduce the errors dramatically and to stabilize the fit. In brief, the procedure can be described as follows:

1. The energy spectrum $W_{l}$ and the wave functions $v^{(l)}$ are accurately determined by measuring the matrix $C_{i j}(t)$ at $t \leqslant t_{\text {unst }}$. We choose $t_{\text {unst }}=5$ for all $L$ and average all $W_{l}$ for $t=$ $t_{0}, \ldots,\left(t_{\text {unst }}-1\right)$. 

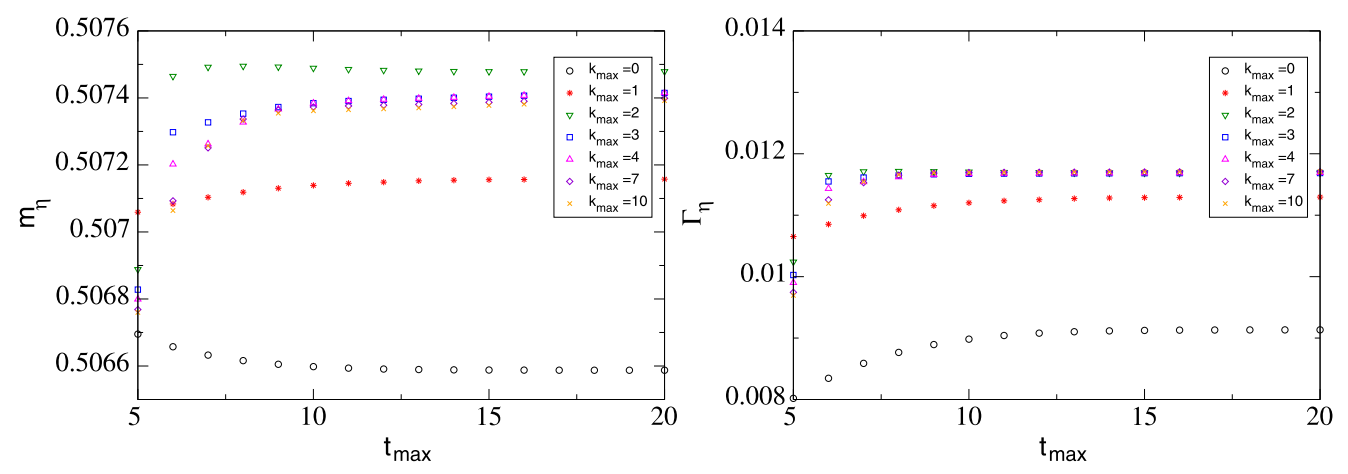

Fig. 3. Checking the convergence of $m_{\eta}$ (left panel) and $\Gamma_{\eta}$ (right panel) against the variation of $t_{\max }$ and $k_{\max }$, for $L=60$. A similar behavior is observed for smaller values of $L$.

2. The function $C_{11}(t)$ is approximated by the multi-exponential function $C_{11}(t)=\sum_{l=1}^{r} z_{l} \times$ $\exp \left(-W_{l} t\right)$, where the $z_{l}=\left|v_{1}^{(l)}\right|^{2}$ are averaged for all $t=t_{0}, \ldots,\left(t_{\text {unst }}-1\right)$. This approximation is used for $t>t_{\text {unst }}$ as well. Note that $z_{l}, l \neq 1$, encode the information about the overlap of $\eta$ and $2 \phi$ states that determines the decay width of a resonance.

3. The expression (23) is fitted to the $C_{11}(t)$ which is approximated by the multi-exponential function.

The MC simulations were carried out for various lattice sizes in the interval $L=24-60$, while the value $T=100$ remained fixed throughout the simulations. We have used bases containing 4-6 operators and performed test runs for some (large) values of $L$ by using the basis of 8 and 10 operators. In the fit, all data between $t=1$ and $t=t_{\max }$ were used. The errors in our results are purely statistical and were estimated by performing 5 independent simulations with $10^{6}$ configurations each. In addition, we find that the increase of the number of operators to 8 or 10 operators does not affect the result within the errors.

First, the stability of our results was checked, when $t_{\max }$ and $k_{\max }$ increase. The result of this check is displayed in Fig. 3, where the dependence of the real and imaginary parts of the resonance pole position on $t_{\max }$ is plotted for different values of $k_{\max }$. It is seen that for $t_{\max } \geqslant$ 10-12 both the energy and the width remain almost constant and converge rapidly in $k_{\max }$ already at $k_{\max }=3$, if the Bayesian fit is performed. The similar behavior is observed at all values of $L$. The final result for the resonance pole parameters is always given at $k_{\max }=10$.

In order to ensure that, performing the Bayesian fit, a bias is not introduced in the extracted values of the resonance parameters, one has to check that there exists a range of the scale parameter $S$ where the energy and the width depend weakly on $S$. The results for both quantities at different values of $L$ look qualitatively similar. In Fig. 4 we present the plot for the width at $L=60$. As seen from Fig. 4 , a wide plateau emerges around $S \simeq 10^{5}$, where the scale dependence practically disappears while the convergence in $k_{\max }$ still persists. This is the window, where the extraction of the width is finally carried out. Increasing $S$ even further, the convergence in $k_{\max }$ breaks down, and the result cannot be trusted any longer.

Finally, since our MC data have been calculated at a finite $L$, whereas the formula (23) refers to the limit $L \rightarrow \infty$, there is an expected residual volume dependence in the parameters $E_{0}, \Gamma$. Fig. 5 displays this dependence. In particular, it is seen that there is a rather strong variation of the width at small values of $L$ that flattens around $L=48$. In the present paper we do not attempt 


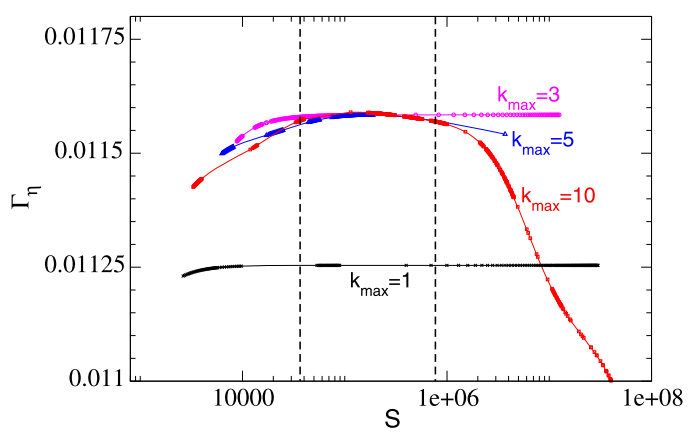

Fig. 4. Dependence of the width on the scale $S$ used in the Bayesian fit at different $k_{\text {max }}$. For small values of $S$, the result is scale-dependent. For large $S$, the result does not converge with $k_{\max }$. There exists a plateau around $S \simeq 10^{5}$ where the procedure converges and yields a scale-independent result.
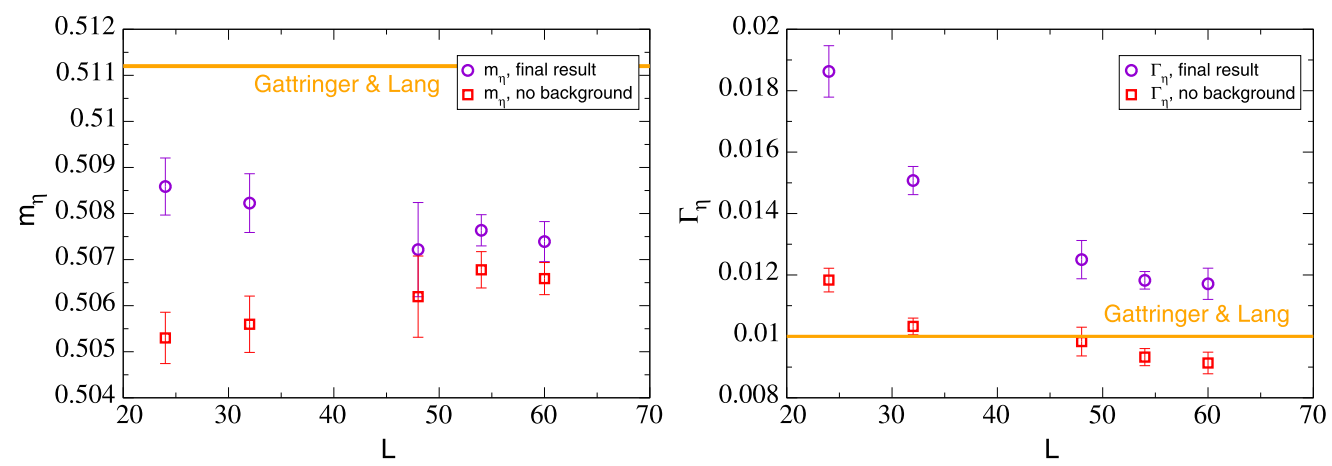

Fig. 5. The energy and the width of the resonance, extracted from the data at $k_{\max }=10$ (final result). For comparison, we display the result at $k_{\max }=0$ (no background) and the result, taken from Refs. [27,28] (the error quoted in these references corresponds to the thickness of the lines). The errors in our calculations are purely statistical.

to quantitatively describe the finite volume artefacts. This issue forms the subject of a separate investigation and we plan to address it in the future.

From Fig. 5 it is also seen that the effect of the background on the real part of the pole position is small, whereas the imaginary part is far more sensitive to it. Namely, the two values of $E_{0}$, calculated at $L=60$ for $k_{\max }=0$ and $k_{\max }=10$ differ by $\simeq 0.2 \%$, whereas the same calculation for the width yields $\Gamma_{\eta}=(0.91 \pm 0.04) \cdot 10^{-2}$ and $\Gamma_{\eta}=(1.17 \pm 0.05) \cdot 10^{-2}$, respectively. In general, one may conclude that the effect of the background cannot be neglected.

The final result for the real and imaginary parts of the pole position (for $L=60$ ) are

$$
\begin{aligned}
& m_{\eta}=2 m_{\phi}+E_{0}=0.5074 \pm 0.0004, \\
& \Gamma_{\eta}=\Gamma=(1.17 \pm 0.05) \cdot 10^{-2}
\end{aligned}
$$

(errors are only statistical). This result can be checked by using the effective-range expansion for the scattering phase (cf. with Ref. [27])

$$
-\frac{p}{W} \tan \delta(p)=a-b p^{2}, \quad W=2 \sqrt{m_{\phi}^{2}+p^{2}},
$$

where the parameters $a$ and $b$ are related to $m_{\eta}$ and $\Gamma_{\eta}$ through 


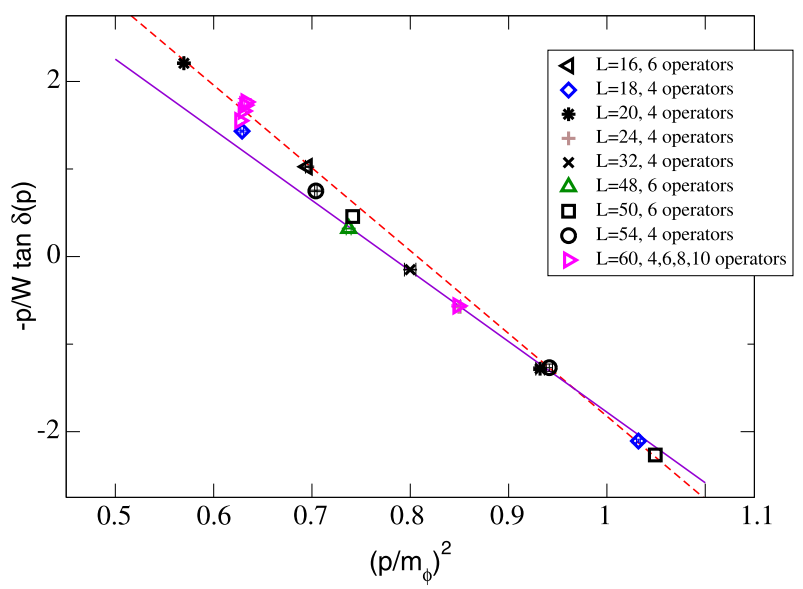

Fig. 6. The quantity $-p / W \tan \delta(p)$, extracted from the energy spectrum, vs. $\left(p / m_{\phi}\right)^{2}$. The solid line is the linear fit $a-b p^{2}$, with $a, b$ determined from Eqs. (35) and (37), using the central values of $m_{\eta}$ and $\Gamma_{\eta}$. To draw the dashed line, we have used $m_{\eta}, \Gamma_{\eta}$ from Ref. [27] instead of Eq. (35) (cf. with Fig. 2 of that paper). The errors in the data are purely statistical. In addition, we have indicated the dimension of the operator basis $O_{j}$ used to extract the spectrum.

$$
m_{\eta}=2 \sqrt{m_{\phi}^{2}+\frac{a}{b}}, \quad \Gamma_{\eta}=\frac{4}{b m_{\eta}^{2}} \sqrt{\frac{a}{b}} .
$$

As one sees from Fig. 6, our phase shift results are generally in agreement with the results of the Ref. [27]. However, since the data are not exactly linear, the question arises, which interval in the variable $p^{2}$ should be used in the fit to determine the coefficients $a$ and $b$. For instance, the extracted values of the phase shift in the vicinity of the resonance $\left(p / m_{\phi}\right)^{2} \simeq 0.8$ neatly follow the straight line with the parameters $a, b$, which were determined from Eqs. (36), using the central values of $m_{\eta}, \Gamma_{\eta}$ in Eq. (35).

Now, we are in a position to compare our results to those of Refs. [27,28]. The difference in the real part of the resonance pole position is small - both results agree with an accuracy of better than one per cent. The effect is larger in the imaginary part. However, one should keep in mind that the magnitude of the imaginary part is approximately 50 times smaller than the real part. As one concludes from Fig. 6, a relatively large effect in the imaginary part could be related, e.g., to the fact that the effective range plot is not exactly linear. Therefore, it seems plausible that the systematic errors both in Refs. [27,28] and in the present paper are underestimated. We expect that the results should agree within the errors.

Note that, once the plateau in $L$ sets in, the energy and the width within our method can be extracted at a single, albeit large value of $L$. This can be related to the fact that our method uses additional input information from MC simulations. In particular, apart from the energy spectrum, the two-point function $C_{11}(t)$ contains the information about the transition matrix elements encoded in the constants $z_{l}, l \neq 1$.

Last but not least, we have also checked that our method works in the non-interacting case as well. Setting $g=0$ and adjusting $\kappa_{\phi}, \kappa_{\eta}$ in the Lagrangian to keep the masses of $\phi$ and $\eta$ the same as in the interacting case (see Refs. [27,28]), we have done the calculation of the function $C_{11}(t)$ anew. The fitted width turns out to be two orders of magnitude smaller as compared to the interacting case. This obviously corresponds to a stable particle. 


\section{Conclusions}

i) In the present paper we have proposed a novel method to extract the resonance pole position on the lattice. The method is based on the universal representation of the two-point function Eq. (23), which is valid given the sole assumption that an isolated low-lying resonance is present in the system. The energy and the width of this resonance are determined from the fit of Eq. (23) to the lattice MC data.

ii) Our method is at present restricted to elastic scattering. It remains to be seen whether such a universal representation can be derived in more complicated cases (e.g., for the multi-channel scattering, see Ref. [38], or in case of the multi-particle final states) as well.

iii) The proposed method provides an alternative to Lüscher's approach to the resonances. In the latter, the volume dependence of the spectrum on moderately large lattices is studied. The spectrum consists of scattering states only - the resonance has already decayed. In our approach the two-point function is studied at finite times $t$ (when the resonance is still "alive") and for the asymptotically large values of $L$.

iv) In certain cases, the numerical accuracy of the method can be improved considerably, if the multi-exponential representation of the two-point function is used in the fit instead of calculating this function directly through MC simulations at all values of $t$. The coefficients of the multi-exponential representation are obtained by solving the generalized eigenvalue problem and, in particular, encode the decay matrix elements.

v) Below we comment on the range of applicability of the method.

The interval of $t$ used in the fit is restricted from below by the first low-lying resonance or inelastic threshold. In order to suppress these high-energy singularities, the condition $\left(\omega_{\mathrm{h}}-\omega_{\min }\right) t_{\min } \gg 1$ should be fulfilled, where $\omega_{\mathrm{h}}$ denotes the CM energy for the lowest high-energy singularity.

The question of the upper bound for $t$ is more subtle. The value of $t$, where the threshold effects start to dominate over the resonance contribution, depends not only on the spectrum but on the matrix elements of the source/sink operators as well. At this stage, we scan the available values of both $t_{\max }$ and $t_{\min }$, in order to establish a window where the final result does not depend on the choice of the fitting interval.

The product $\Gamma t$ within the fitting interval should be of order of unity. If this product is much larger than unity, the resonance will immediately decay. On the contrary, if this quantity is very small, the resonance behaves pretty much like a bound state and an accurate determination of the width can be difficult. In the $1+1$-dimensional example that we have considered here, this quantity was approximately equal to 0.1 .

If one extracts the parameters of a resonance, fitting the infinite-volume ansatz to the data taken at a finite volume, the fit results will contain finite-volume artefacts. From Fig. 5 one may conclude that these artefacts can be quite sizable. At this stage, it is very hard to say, how large $L$ should be to render these artefacts small. For example, in the $1+1$-dimensional Ising model at $L=48$ the plateau starts to set in. We are, however, still lacking an a priori estimate. Moreover, it would be very interesting to work out an analytic expression for the artefacts which may be useful in the analysis of the data taken on not-so-large lattices. Further, using asymmetric lattices (as in Ref. [13]) could be advantageous in this context. These issues, however, form a separate subject of investigation.

vi) Recently, the excited meson and baryon spectra have been determined by several lattice collaborations using the generalized eigenvalue equation (see, e.g. Refs. [19-21] for the latest work on the subject). These calculations closely resemble the calculations in the $1+1$ - 
dimensional toy model, which were presented in this paper. In our opinion, it would be very interesting to apply the proposed method to the data and if possible try to locate the resonance pole(s). This can be done at no additional cost, since the results of already existing MC simulations would be used.

\section{Acknowledgements}

The authors are thankful to Jürg Gasser for a close and fruitful cooperation at all stages of the work on the project. We wish to thank Ferenc Niedermayer for his constant readiness to help, for numerous discussions and suggestions. We also thank R.G. Edwards, C. Gattringer, M. Göckeler, P. Hasenfratz, C. Lang, D. Lee, C. McNeile, C. Michael, D. Phillips, M. Schindler, K. Urbach and U. Wenger for interesting discussions. Work supported in part by DFG (SFB/TR 16, "Subnuclear Structure of Matter"), by the Helmholtz Association through funds provided to the virtual institute "Spin and strong QCD" (VH-VI-231) and by COSY FFE under contract 41821485 (COSY 106). We also acknowledge the support of the European Community-Research Infrastructure Integrating Activity "Study of Strongly Interacting Matter" (acronym HadronPhysics2, Grant Agreement No. 227431) under the Seventh Framework Programme of EU. A.R. acknowledges financial support of the Georgia National Science Foundation (Grant \#GNSF/ST08/4-401).

\section{Appendix A. Continuum limit in the matrix elements}

The regular summation theorem [30], which is used in order to perform the continuum limit in the sums over the discrete momentum eigenvalues, implies that the integrand is a continuous function in the momenta. However, the finite-volume matrix elements $\langle 0|\phi(0)| \beta\rangle$, which enter Eq. (8), contain Lüscher's zeta-function and can become singular. Here, for one particular example, we shall demonstrate how these singularities are lifted.

The averaged quantities, for which the validity of the regular summation theorem will be checked, are defined in Eq. (14). From now on, without loss of generality, we shall work in the center-of-mass frame $\mathbf{k}=0$. We wish to demonstrate that

$$
\lim _{L \rightarrow \infty} A\left(\omega_{0}, \Delta, \mathbf{0}\right)=A^{\infty}\left(\omega_{0}, \Delta, \mathbf{0}\right), \quad \omega_{0}>\omega_{\min }, \Delta>0 .
$$

More precisely, the difference between the both sides of the above equation vanishes faster that any negative power of $L$, as $L \rightarrow \infty$.

Let $\omega_{0}$ be in the elastic scattering region. Since $L$ is large, characteristic momenta are small and non-relativistic quantum mechanics provides an adequate description of a problem under consideration. Let us consider two massive (distinguishable) particles in the CM frame. The state vector corresponding to the eigenvalue $E_{\beta}$ is given by

$$
|\beta\rangle=\frac{1}{L^{d / 2}} \sum_{\mathbf{q}} f_{\beta}(\mathbf{q})|\mathbf{q},-\mathbf{q}\rangle, \quad|\mathbf{q},-\mathbf{q}\rangle=a_{1}^{\dagger}(\mathbf{q}) a_{2}^{\dagger}(-\mathbf{q})|0\rangle,
$$

where $a_{i}^{\dagger}, i=1,2$ denote the creation operators for the particles 1 and 2, respectively, and the wave function $f_{\beta}(\mathbf{q})$ is normalized, according to

$$
\langle\beta \mid \beta\rangle=\frac{1}{L^{d}} \sum_{\mathbf{q}}\left|f_{\beta}(\mathbf{q})\right|^{2}=1 .
$$


For simplicity, let us further assume that the interaction between the particles is described by a separable potential $V(\mathbf{p}, \mathbf{k})=g v(\mathbf{p}) v(\mathbf{k})$, where the function $v(\mathbf{p})$ corresponds to a smooth cutoff at large momenta. Note that in the following we will never need the explicit form of this function. The $T$-matrix is given by

$$
T(\mathbf{p}, \mathbf{q} ; E)=\frac{v(\mathbf{p}) v(\mathbf{q})}{g^{-1}-I(E)}, \quad I(E)=\frac{1}{L^{d}} \sum_{\mathbf{k}} \frac{v^{2}(\mathbf{k})}{\mathbf{k}^{2}-k_{0}^{2}}, k_{0}^{2}=2 \mu E,
$$

where $\mu$ denotes the reduced mass of the system.

In the limit $E \rightarrow E_{\beta}$ the $T$-matrix has a pole. In the vicinity of the pole, it behaves as

$$
T(\mathbf{p}, \mathbf{q} ; E)=\frac{v(\mathbf{p}) v(\mathbf{q})}{g^{-1}-I\left(E_{\beta}\right)-I^{\prime}\left(E_{\beta}\right)\left(E-E_{\beta}\right)+\cdots}=\frac{v(\mathbf{p}) v(\mathbf{q})}{-I^{\prime}\left(E_{\beta}\right)\left(E-E_{\beta}\right)+\cdots} .
$$

From this expression, we may read off the wave function corresponding to the eigenvalue $E_{\beta}$

$$
f_{\beta}(\mathbf{q})=\sqrt{\frac{2 \mu}{I^{\prime}\left(E_{\beta}\right)}} \frac{v(\mathbf{q})}{\mathbf{q}^{2}-k_{0 \beta}^{2}}, \quad k_{0 \beta}^{2}=2 \mu E_{\beta} .
$$

The normalization of this wave function was chosen so that $f_{\beta}(\mathbf{p})$ obeys Eq. (A.3).

Take the composite field $\phi(0)=\phi_{1}(0) \phi_{2}(0)$, where the $\phi_{i}(0), i=1,2$, denote the elementary particle fields. In momentum space,

$$
\phi_{i}(0, \mathbf{x})=\frac{1}{L^{d / 2}} \sum_{\mathbf{k}} \mathrm{e}^{i \mathbf{k x}} a_{i}(\mathbf{k}) .
$$

The matrix element that enters the spectral function is given by

$$
\langle 0|\phi(0)| \beta\rangle=\frac{1}{L^{3 d / 2}} \sum_{\mathbf{q}} f_{\beta}(\mathbf{q}) \doteq \frac{1}{L^{d}} \tilde{f}_{\beta}(\mathbf{0}),
$$

where $\tilde{f}_{\beta}(\mathbf{r})$ denotes the Fourier-transform of $f_{\beta}(\mathbf{q})$. The averaged spectral function is written in the following form

$$
A\left(\omega_{0}, \Delta, \mathbf{0}\right)=\frac{1}{L^{d}} \sum_{\beta} \theta\left(\omega_{0}+\Delta / 2-E_{\beta}\right) \theta\left(E_{\beta}-\omega_{0}+\Delta / 2\right)\left|\tilde{f}_{\beta}(\mathbf{0})\right|^{2} .
$$

Hence, in order to verify the applicability of the regular summation theorem in this case, it suffices to show that $\tilde{f}_{\beta}(\mathbf{0})$ is a regular function of $k_{0 \beta}$. As anticipated, this function contains Lüscher's zeta-function which is singular at $k_{0 \beta}^{2}=(2 \pi \mathbf{n})^{2} / L^{2}$. However, the factor $I^{\prime}\left(E_{\beta}\right)$, which enters the normalization, contains the zeta-function as well. It is easy to check that the singular factors in the numerator and the denominator cancel, and the regular summation theorem holds.

\section{Appendix B. Emergence of the second Riemann sheet in the infinite volume limit}

Let us consider ${ }^{7}$ the function $F_{L}$ of the complex variable $z$

\footnotetext{
7 We are indebted to Jürg Gasser who indicated this example to us.
} 


$$
F_{L}(z)=\frac{1}{1-z+\epsilon^{2} \sqrt{z} \cot (\sqrt{z} L)}, \quad z \in \mathbb{C} .
$$

Note that this function resembles the propagator of an unstable particle in the $1+1$-dimensional effective field theory [5]. Further, it can be shown that

$$
\operatorname{sign}(\operatorname{Im}(\sqrt{z} \cot (\sqrt{z} L)))=-\operatorname{sign}(\operatorname{Im}(z)) .
$$

According to this condition, the denominator cannot vanish outside the real axis. Thus, the only singularities of $F_{L}(z)$ are simple poles on the positive real axis.

If $\operatorname{Im}(z) \neq 0$, in the limit $L \rightarrow \infty$ we have $\cot (\sqrt{z} L) \rightarrow-i \operatorname{sign}(\operatorname{Im}(z))$ and, therefore,

$$
F_{\infty}(z)=\frac{1}{1-z+\epsilon^{2} \sqrt{-z}} .
$$

The difference $\left|F_{L}(z)-F_{\infty}(z)\right|$ vanishes exponentially with $L$, if $\operatorname{Im}(z) \neq 0$. Note that, unlike $F_{L}(z)$, which is a meromorphic function, $F_{\infty}(z)$ is analytic in the complex plane cut along the positive real axis. This is what is meant when we speak of "the poles merging into the cut."

Moreover, the function $F_{\infty}(z)$ has a couple of complex-conjugated poles on the second Riemann sheet. These poles are solutions of the equation $1-z+\epsilon^{2} \sqrt{-z}$ that gives $z_{ \pm}=$ $1 \mp i \epsilon^{2}+O\left(\epsilon^{4}\right)$. If $\epsilon^{2}$ is small, these poles come close to the physical scattering region and influence $F_{\infty}(z)$ on the physical sheet. Since away from the real axis the difference between $F_{\infty}(z)$ and $F_{L}(z)$ vanishes exponentially at a large $L$, the effect of the poles on the second Riemann sheet is felt in $F_{L}(z)$ as well.

\section{References}

[1] M. Lüscher, DESY-88-156 Lectures given at Summer School 'Fields, Strings and Critical Phenomena', Les Houches, France, 28 June-5 August 1988.

[2] U.-J. Wiese, Nucl. Phys. B (Proc. Suppl.) 9 (1989) 609.

[3] M. Lüscher, Nucl. Phys. B 354 (1991) 531.

[4] M. Lüscher, Nucl. Phys. B 364 (1991) 237.

[5] D. Hoja, U.-G. Meißner, A. Rusetsky, arXiv:1001.1641 [hep-lat].

[6] M. Göckeler, H.A. Kastrup, J. Westphalen, F. Zimmermann, Nucl. Phys. B 425 (1994) 413, arXiv:hep-lat/9402011.

[7] S. Aokim, et al., CP-PACS Collaboration, Phys. Rev. D 76 (2007) 094506, arXiv:0708.3705 [hep-lat].

[8] M. Göckeler, R. Horsley, Y. Nakamura, D. Pleiter, P.E.L. Rakow, G. Schierholz, J. Zanotti, QCDSF Collaboration, PoS LATTICE2008 (2008) 136, arXiv:0810.5337 [hep-lat].

[9] K. Rummukainen, S.A. Gottlieb, Nucl. Phys. B 450 (1995) 397, arXiv:hep-lat/9503028.

[10] M. Asakawa, T. Hatsuda, Y. Nakahara, arXiv:hep-lat/0011040v2.

[11] S. Sasaki, K. Sasaki, T. Hatsuda, M. Asakawa, Nucl. Phys. B (Proc. Suppl.) 119 (2003) 302, arXiv:hep-lat/0209059.

[12] K. Sasaki, S. Sasaki, T. Hatsuda, Phys. Lett. B 623 (2005) 208, arXiv:hep-lat/0504020.

[13] C. Michael, Nucl. Phys. B 327 (1989) 515.

[14] R.D. Loft, T.A. DeGrand, Phys. Rev. D 39 (1989) 2692.

[15] L. Lellouch, M. Lüscher, Commun. Math. Phys. 219 (2001) 31, arXiv:hep-lat/0003023.

[16] J.J. Dudek, R.G. Edwards, M.J. Peardon, D.G. Richards, C.E. Thomas, Phys. Rev. Lett. 103 (2009) 262001, arXiv:0909.0200 [hep-ph].

[17] S. Cohen, et al., arXiv:0911.3373 [hep-lat].

[18] J.M. Bulava, et al., Phys. Rev. D 79 (2009) 034505, arXiv:0901.0027 [hep-lat].

[19] J. Bulava, et al., arXiv:1004.5072 [hep-lat].

[20] J.J. Dudek, R.G. Edwards, M.J. Peardon, D.G. Richards, C.E. Thomas, arXiv:1004.4930 [hep-ph].

[21] G.P. Engel, C.B. Lang, M. Limmer, D. Mohler, A. Schafer, BGR [Bern-Graz-Regensburg] Collaboration, arXiv: 1005.1748 [hep-lat].

[22] A. Walker-Loud, et al., Phys. Rev. D 79 (2009) 054502, arXiv:0806.4549 [hep-lat].

[23] T. Burch, C. Gattringer, L.Y. Glozman, C. Hagen, D. Hierl, C.B. Lang, A. Schafer, Phys. Rev. D 74 (2006) 014504, arXiv:hep-lat/0604019. 
[24] C. Alexandrou, A. Tsapalis, Phys. Rev. D 73 (2006) 014507, arXiv:hep-lat/0503013.

[25] C. Alexandrou, A. Tsapalis, PoS LAT2005 (2006) 023, arXiv:hep-lat/0509139.

[26] Z.Y. Niu, M. Gong, C. Liu, Y. Shen, Phys. Rev. D 80 (2009) 114509, arXiv:0909.3154 [hep-lat].

[27] C.R. Gattringer, C.B. Lang, Phys. Lett. B 274 (1992) 92.

[28] C.R. Gattringer, C.B. Lang, Nucl. Phys. B 391 (1993) 463, arXiv:hep-lat/9206004.

[29] B.S. DeWitt, Phys. Rev. 103 (1956) 1565.

[30] M. Luscher, Commun. Math. Phys. 105 (1986) 153.

[31] A. Nolan, M. Peardon, A.O. Cais, PoS LAT2007 (2007) 127.

[32] T.R. Hemmert, B.R. Holstein, J. Kambor, J. Phys. G 24 (1998) 1831, arXiv:hep-ph/9712496.

[33] V. Bernard, U.-G. Meißner, A. Rusetsky, Nucl. Phys. B 788 (2008) 1, arXiv:hep-lat/0702012.

[34] V. Bernard, D. Hoja, U.-G. Meißner, A. Rusetsky, JHEP 0906 (2009) 061m, arXiv:0902.2346 [hep-lat].

[35] H. Krebs, E. Epelbaum, U.-G. Meißner, Phys. Lett. B 683 (2010) 222, arXiv:0905.2744 [hep-th].

[36] M.R. Schindler, D.R. Phillips, Ann. Phys. 324 (2009) 682, arXiv:0808.3643 [hep-ph];

M.R. Schindler, D.R. Phillips, Ann. Phys. 324 (2009) 2051 (Erratum).

[37] R.H. Swendsen, J.-S. Wang, Phys. Rev. Lett. 58 (1987) 86.

[38] M. Lage, U.-G. Meißner, A. Rusetsky, Phys. Lett. B 681 (2009) 439, arXiv:0905.0069 [hep-lat]. 